\title{
The joy of secants
}

\section{Trigonometric Delights}

by Eli Maor

Princeton University Press: 1998. Pp. 236.

$\$ 24.95, £ 17.95$

Geometry Civilized: History, Culture, and Technique

by J. L. Heilbron

Oxford University Press: 1998. Pp. 309.

$\mathfrak{E 3 5 , \$ 6 5}$

\section{Jeremy Gray}

Mathematics in general, and geometry in particular, seem to their practitioners to be both essential and attractive aspects of life. Not so to many who struggled with them at school; and still less, one must presume, do today's school students value them, because they have almost lapsed from the syllabus. How to bridge this fissure?

These two books, which cover geometry and trigonometry, offer an interesting pair of attempts. As their titles suggest, John Heilbron's is the more substantial, and weaves in and out of a delight in the mathematics and a sensitive attention to the variety of cultural occasions for it. The book by Eli Maor is similar, but is lighter culturally and deeper mathematically. But both share a belief that doing the mathematics - solving problems - is part of the fun; both trace the origins of these ideas to a variety of cultures; and both glory in the achievements that geometry and trigonometry have made possible. Any reader not frightened of mathematics should be able to share that emotion and come away with a deeper appreciation of these subjects.

Heilbron's book has an unconventional organization that contributes greatly to its charm. Lofty themes, such as cathedral windows, and aesthetic gems, such as Islamic designs, are mixed with difficult (and sometimes perhaps contrived) exercises in a survey of real and imaginary fields. The exercises are culled from such sources as Egyptian papyri, The Ladies' Diary and textbooks down the ages; and Heilbron adds many of his own. They occur on almost every page, not hived off at the end where they can be skipped; reader, do them! Their effect is to humanize the matter, to connect the important with the mundane, and to suggest that only by mastering these challenges could geometers and architects have accomplished their greatest work.

Equally, the mixture of the elevated and the irritating makes the 'mere' problems more interesting, without implying that they are more significant in themselves. Modern, especially American, calculus textbooks like to insinuate that the subject is 'really' useful by similar devices, but they fail because the examples are all too often artificial questions dressed up to look like real ones. Heilbron

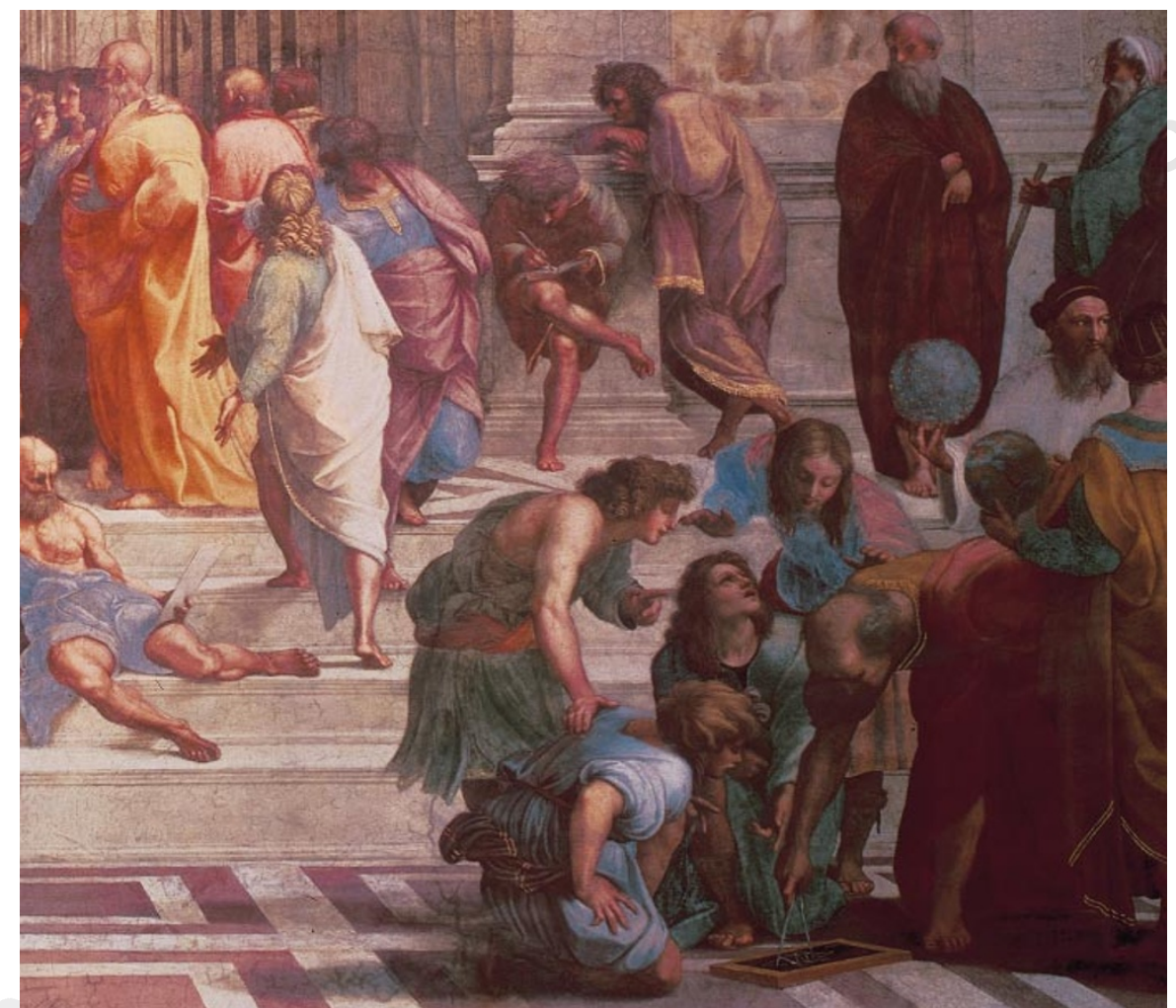

Polygon with polymaths: Euclid and his students construct a star hexagon, in this detail from Raphael's fresco The School of Athens.

succeeds because of the wealth of his examples. Surely, one comes to realize, this is how the old experts saw the subject: as a real mix of the sublime and the fiddly. Designing a large Gothic window and working out an inelegant trigonometric problem are part of the same activity, which a mathematician achieves by doing the sums.

The book is wonderfully illustrated. There are diagrams on almost every page, apt illustrations from older books, and well chosen photographs, together with eight colour plates. The appearance of the book is quite seductive, for which Oxford University Press should be congratulated.

Heilbron covers Euclidean geometry, including the behaviour of circles, and plane trigonometry. All is explained from scratch, but not in the style of a textbook. Nor is it exactly a history of these topics, although it is erudite. The effect is to impart dignity to the subject, and wisdom to its readers.

Maor's book is smaller and lighter, but none the worse for that. It includes topics drawn from the calculus of the trigonometric functions: infinite series, the hyperbolic trigonometric functions, and complex numbers. It is more expository than Heilbron's book, with fewer exercises, and is more conventionally organized as a history. Topics include Ptolemy's tables, the Hindu origin of the trigonometric functions, trigonometric identities, the shape of the Earth, cartography, de Moivre's formula and its consequences, and rigour in analysis. Little of this is

Students of trigonometry and their teachers will enjoy finding the core syllabus so refreshingly opened up. If they find the accounts, especially towards the end of the book, to be tantalizingly short, this is all the more reason to emulate the author and find out more for themselves, and Maor gives advice on how this can be done.

Both authors suggest that geometry and trigonometry were casualties of the 'new maths' reforms of the 1960s, and that the calculator-driven education of today is no better. The case for these subjects, at a genuinely elementary level, based on their utility and beauty, has seldom been better put. Heilbron's, in particular, is a deeply humanist work, which is a strange thing to say about a book with detailed geometry on every page. It may be that the devil is in the details, but so too is the step-by-step, line by painful line, progress of human achievement. The full immersion in the subject that Heilbron provides allows one to recognize oneself in the work of others, and to share in that inclusive web of our history, culture and technique. $\square$ Jeremy Gray is in the Faculty of Mathematics and Computing, The Open University, Milton Keynes MK7 6AA, UK. new, but the mixture is engagingly presented. 